\title{
Oscillations of sterile neutrinos from dark matter decay eliminates the IceCube-Fermi tension
}

\author{
Luis A. Anchordoqui, ${ }^{1,2,3}$ Vernon Barger, ${ }^{4}$ Danny Marfatia, ${ }^{5}$ Mary Hall Reno, ${ }^{6}$ and Thomas J. Weiler ${ }^{7}$ \\ ${ }^{1}$ Department of Physics and Astronomy, Lehman College, City University of New York, NY 10468, USA \\ ${ }^{2}$ Department of Physics, Graduate Center, City University of New York, NY 10016, USA \\ ${ }^{3}$ Department of Astrophysics, American Museum of Natural History, NY 10024, USA \\ ${ }^{4}$ Department of Physics, University of Wisconsin, Madison, WI 53706, USA \\ ${ }^{5}$ Department of Physics and Astronomy, University of Hawaii, Honolulu, HI 96822, USA \\ ${ }^{6}$ Department of Physics and Astronomy, University of Iowa, Iowa City, IA 52242, USA \\ ${ }^{7}$ Department of Physics and Astronomy, Vanderbilt University, Nashville TN 37235, USA
}

\begin{abstract}
IceCube has observed a flux of cosmic neutrinos, with a "bump" in the energy range $10 \lesssim E / \mathrm{TeV} \lesssim 100$ that creates a $3 \sigma$ tension with $\gamma$-ray data from the Fermi satellite. This has been interpreted as evidence for a population of hidden cosmic-ray accelerators. We propose an alternative explanation of this conundrum on the basis of cold dark matter which decays into sterile neutrinos that after oscillations produce the bump in the cosmic neutrino spectrum.
\end{abstract}

\section{INTRODUCTION}

The most immediate message emerging from IceCube's discovery of cosmic neutrinos is that the flux level observed is exceptionally high by astronomical standards [1--3]. The magnitude of the observed diffuse neutrino flux is above about the level of the WaxmanBahcall bound [4], which applies to neutrino production in optically thin sources. As always, the devil is in the details. Neutrinos are inevitably produced in association with gamma rays when accelerated baryonic cosmic rays produce charged and neutral pions in interactions with intense radiation fields or dense clouds of gas surrounding the accelerator. The subsequent decay of charged pions via $\pi^{+} \rightarrow \mu^{+} v_{\mu}$ followed by muon decay $\mu^{+} \rightarrow e^{+} v_{e} \bar{v}_{\mu}$ (and the charge-conjugate processes) produce a neutrino flux, whereas the associated gamma-ray flux originates in the decay of neutral pions, $\pi^{0} \rightarrow \gamma \gamma$. On average, pionic $v^{\prime}$ s and $\gamma^{\prime}$ s carry one quarter and one half of the energy of the parent pion, respectively. If the sources were optically thin, then on the basis of these approximations we would expect roughly equal fluxes of $v^{\prime} \mathrm{s}$ and $\gamma^{\prime} \mathrm{s}$ [5]. Before confronting this equality with experiment, we must account for the fact that, unlike neutrinos, gamma rays are degraded in energy by electromagnetic cascades and contribute to the diffuse $\mathrm{GeV}-\mathrm{TeV}$ flux that has been precisely measured by the Fermi satellite [6]. The neutrino flux observed by IceCube in the energy range $10 \lesssim E / \mathrm{TeV} \lesssim 100$ creates a $3 \sigma$ tension with Fermi data where there isn't a commensurate gamma ray flux.

The IceCube-Fermi tension has been interpreted as evidence for a population of hidden cosmic-ray accelerators, viz. sources that are more efficient neutrino than gamma-ray emitters [7-9]. This interpretation, however, requires some fine-tuning as it would need a source environment with:

- a low-density region for cosmic ray protons to be accelerated without suffering catastrophic spallations;

- a medium-density region where the baryonic cos- mic rays can interact, but charged pions are able to decay;

- a high-density region in the outer parts of the source to trap the photons, and perhaps also the baryonic cosmic rays.

Since the proton-proton cross section is comparable to the pion-proton cross section $\left(\sigma_{\pi p} / \sigma_{p p} \simeq 2 / 3\right)$, the first two requirements are difficult to reconcile. Neutrino production requires an optically thin source: the high energy cutoff of the cosmic ray spectrum comes primarily from pion production, however, pion production cannot be significant at lower energies to allow cosmic rays to be adequately accelerated. While protons experience magnetic confinement, neutrons can escape, and then decay to yield a cosmic ray proton flux.

These considerations can be translated to conditions on the characteristic time scales: the proton interaction time scale $\tau_{\text {int }}$, the neutron decay lifetime $\tau_{n}$, the cycle time of confinement $\tau_{\text {cycle, }}$ and the total proton confinement time $\tau_{\text {conf }}$. For sufficient acceleration, $\tau_{\text {int }} \gg \tau_{\text {cycle }}$ is required. Additionally, in order for neutrons to escape the source, $\tau_{\mathrm{n}}>\tau_{\text {cycle }}$. Finally, to produce neutrons and neutrinos, $\tau_{\text {int }} \ll \tau_{\text {conf }}$ [10]. These conditions are required of optically thin sources. A condition for hidden cosmic-ray accelerators is $\tau_{\mathrm{n}}<\tau_{\text {cycle, }}$ which contradicts the conditions for neutrino emission from optically thin sources. Instead, to trap gamma rays (produced via $\pi^{0}$ decay) and cosmic rays, one would need a high-density optically thick region in the outer parts of the source to encircle the optically thin engine. This hypothetical source structure is certainly not the most natural and it needs some fine-tuning.

In this paper we present an alternative explanation of the IceCube-Fermi tension in which active neutrinos originate via decaying dark matter into sterile neutrinos. These sterile neutrinos oscillate to produce the "bumpy" signal in the $10 \lesssim E / \mathrm{TeV} \lesssim 100$ energy range. The dark matter origin of IceCube neutrinos has been extensively discussed in the literature. However, in most of these studies the dark matter particle couples to the Standard 
Model (SM) through: (i) the quark $Q$ doublet, (ii) the lepton $L$ and/or Higgs $H$ doublets, (iii) the $W$ or $Z$ gauge bosons, (iv) a massless dark photon which mixes with the ordinary photon, or $(v)$ a dark $Z^{\prime}$ which mixes with the $Z$ [11-21]. Therefore, in these models, dark matter decay leads to fluxes of both neutrinos and photons. As a matter of fact, the photon signal has been used to constrain these scenarios [22]-24]. The novelty of the scenario proposed herein is that the dominant flux of IceCube neutrinos in the energy range $10 \lesssim E / \mathrm{TeV} \lesssim 100$ originates via oscillations of sterile neutrinos produced at cosmological distances, but without a gamma-ray counterpart, thereby addressing the IceCube-Fermi tension; for earlier work that explains the IceCube $\mathrm{PeV}$ events without consideration of Fermi data see [25].

The layout of the paper is as follows. We begin in Sec.II by reviewing the cosmological constraints on decaying dark matter. In Sec. IIII we provide an outline of the basic setup of the Boltzmann transport equation, specifying model assumptions on the neutrino mass spectrum, sterile neutrino interactions, and oscillation parameters. In Sec. [V] we confront the model predictions with IceCube data. In Sec. $\mathrm{V}$ we discuss related phenomenology. Finally, we summarize our results and draw our conclusions in Sec.VI

\section{CONSTRAINTS ON DECAYING DARK MATTER}

A large number of observations in cosmology and astrophysics provide overwhelming evidence for dark matter [26]. However, so far the origin of this evidence has been purely gravitational; hence we have very few clues about the particle nature of the dark matter. We know dark matter is essential for structure formation in the late universe, so most of it (though not all) must be stable on cosmological time scales.

The dominant paradigm in dark-matter phenomenology has been to consider frameworks in which the total dark matter (DM) density today, $\Omega_{\mathrm{dm}}$, is made up of one stable particle species. However, it may well be that many particle species - perhaps even a vast number contribute nontrivially to the abundance, with some of these quasi-stable, as in Ref. [27].

For simplicity, we consider two cold components to constitute dark matter, one of which is stable, denoted by $\chi_{\mathrm{sc}}$, and the other which decays, denoted by $\chi_{\mathrm{dc}}$, with a lifetime $\tau_{\mathrm{dc}} \gtrsim t_{\mathrm{LS}}$, where $t_{\mathrm{LS}}$ is the time of last scattering. The fraction $F$ of decaying dark matter is

$$
F \equiv \frac{\Omega_{\mathrm{dc}}}{\Omega_{\mathrm{dm}}},
$$

where $\Omega_{\mathrm{dm}}=\Omega_{\mathrm{dc}}+\Omega_{\mathrm{sc}}$, and the $\Omega^{\prime}$ s are the energy densities today in units of the critical density. Here, $\Omega_{\mathrm{dc}}$ is the density parameter today as if none of it had decayed.

We consider the particular case in which $\chi_{\mathrm{dc}}$ decays with a branching fraction of essentially unity via $\chi_{\mathrm{dc}} \rightarrow v_{s} \bar{v}_{s}$, where $v_{s}$ is a sterile neutrino that behaves as dark radiation. See Ref. [25] for a model that realizes this scenario. The dynamics associated with the energy of the $v_{s}$ could change the evolution of cosmological perturbations, leading to observable consequences, thereby allowing us to place constraints on $F$ and the decay width $\Gamma_{\mathrm{dc}}$ of $\chi_{\mathrm{dc}}$. Anisotropies in the cosmic microwave background $(\mathrm{CMB})$ temperature and polarization fields measured by the Planck mission [28] when combined with Baryonic Acoustic Oscillations data from BOSS data release 12 (aka DR-12) [29] imply

$$
F<1.28 \times 10^{-2}
$$

and

$$
F \Gamma_{\mathrm{dc}}<2.25 \times 10^{-5} \mathrm{Gyr}^{-1}
$$

at the 95\% CL [30]. This small fraction of dark radiation cannot alter significantly the expansion rate [31-38]. Therefore, we can safely assume that the evolution of the universe is described by the standard $\Lambda$ cold dark matter cosmological model, with the Hubble parameter given by

$$
H(z)=H_{0} \sqrt{\Omega_{\Lambda}+\Omega_{m}(1+z)^{3}+\Omega_{r}(1+z)^{4}},
$$

where $H_{0}=100 \mathrm{~h} \mathrm{~km} / \mathrm{s} / \mathrm{Mpc}$ is the Hubble constant and $\Omega_{\Lambda}, \Omega_{m}$, and $\Omega_{r}$ are the present day fractions of the dark energy density, the non-relativistic matter density, and the radiation density. The cosmological parameters are normalized to CMB data, with $h=0.6766$ [28].

\section{BOLTZMANN TRANSPORT}

The evolution of the neutrino density in phase space is driven by Boltzmann's transport equation, which equates the directional derivative of the distribution function $f$ along the phase flow to a collision integral. Strictly speaking, the change in $f$ along a phase space trajectory with affine parameter $\lambda$ is equal to the phase space density $C[f]$ of point-like collisions that add or remove neutrinos from the trajectory:

$$
\frac{d f}{d \lambda}=C[f]
$$

The phase space measure is defined in such a way that $f$ and $C[f]$ are both invariant scalars. For practical computations, it is necessary to introduce phase space coordinates, which we take to be spacetime position coordinates $x^{\mu}$ and momentum space coordinates $P^{\mu}$. Then,

$$
\left(\frac{d x^{\mu}}{d \lambda} \frac{\partial}{\partial x^{\mu}}+\frac{d P^{\mu}}{d \lambda} \frac{\partial}{\partial P^{\mu}}\right) f\left(x^{\mu}, P^{\mu}\right)=C[f] .
$$

Before proceeding, we pause to present our notation. We adopt lower-case Greek letters from the middle of the alphabet for spacetime components (where indices take on 
values $0,1,2,3)$, lower-case Latin letters from the middle of the alphabet for spatial components (where indices take on values 1,2,3), lower-case Greek letters from the beginning of the alphabet for neutrino flavors (where indices take on value $e, \mu, \tau, s$ ), and lower-case Latin letters from the beginning of the alphabet for neutrino mass eigenstates (where indices take on values 1,2,3,4). Geometry comes into play via the geodesic equation specifying the neutrino trajectories:

$$
\frac{d P^{\mu}}{d \lambda}+\Gamma_{v \sigma}^{\mu} P^{v} P^{\sigma}=0
$$

where

$$
\Gamma_{v \sigma}^{\mu}=\frac{1}{2} g^{\mu \eta}\left(\frac{\partial g_{\sigma \eta}}{\partial x^{v}}+\frac{\partial g_{v \eta}}{\partial x^{\sigma}}-\frac{\partial g_{v \sigma}}{\partial x^{\eta}}\right)
$$

are the affine connection coefficients,

$$
P^{\mu} \equiv \frac{d x^{\mu}}{d \lambda}
$$

is the four-momentum, and $g_{\mu \nu}$ is the metric tensor. Substituting (7) and (9) into (6) we obtain

$$
\left(P^{\mu} \frac{\partial}{\partial x^{\mu}}-\Gamma_{\mu v}^{\sigma} P^{\mu} P^{v} \frac{\partial}{\partial P^{\mu}}\right) f\left(x^{\mu}, P^{\mu}\right)=C[f] .
$$

The pre and post collisional momentum four-vectors are connected by energy-momentum conservation. However, it has long been known that active neutrino interactions on the cosmic neutrino background can be safely neglected [39]. For simplicity, hereafter we assume that sterile neutrino interactions can also be neglected. With this in mind, we consider a gas of collision-free particles.

After production, the sterile neutrinos $v_{s}$ travel over cosmological distances before their arrival at Earth. The flavor composition at Earth is altered by neutrino oscillations, which are due to each neutrino flavor state being a superposition of propagation states $v_{a}$,

$$
\left|v_{\alpha}\right\rangle=\sum_{\alpha} U_{\alpha a}^{*}\left|v_{a}\right\rangle,
$$

where $U_{\alpha a}$ is an element of the mixing matrix $\mathbb{U}$ that connects the flavor and propagation states [40]. For the $3+1$ scenario under consideration $\mathbb{U}$ is a $4 \times 4$ unitary mixing with 16 degrees of freedom: 6 mixing angles and 3 Dirac phases [41-44]. Unitarity ensures conservation of the total number of neutrinos of all flavors. Transitions from flavor $\left|v_{\alpha}\right\rangle$ to $\left|v_{\beta}\right\rangle$ (or from $\left|\bar{v}_{\alpha}\right\rangle$ to $\left|\bar{v}_{\beta}\right\rangle$ ) can only be described by their oscillation-averaged transition probability, which is found to be $P_{\alpha \beta}=\sum_{a}\left|U_{\alpha a}\right|^{2}\left|U_{\beta a}\right|^{2}$ [45]. All in all, the neutrino fluxes of different flavors at IceCube is given by

$$
\left.\frac{d \Phi_{v_{\alpha}}}{d E}\right|_{\oplus}=P_{\alpha s} \frac{d \Phi_{v_{s}}}{d E}=\sum_{a}\left|U_{\alpha a}\right|^{2}\left|U_{s a}\right|^{2} \frac{d \Phi_{v_{s}}}{d E}
$$

after all terms depending on mass squared differences are averaged out over cosmological distances. Assuming a general unitary mixing in the $3+1$ flavor scenario such that the mixing parameters relevant for high-energy neutrinos are unbounded [44], Monte Carlo samples show that for a source of $100 \%$ sterile neutrinos there is a maximum of $75 \%$ transformed into active flavors after oscillations, i.e., one is left with a minimum of $25 \%$ sterile neutrinos at Earth [46]. The effective fraction of active neutrinos on Earth resulting from oscillations of sterile neutrinos will be taken as a free parameter of the model, but constrained to be less than $75 \%$.

For each neutrino mass state $m_{a}$, we identify $\lambda=\tau_{a} / m_{a}$, where $\tau_{a}$ is the proper time. The norm of the fourmomentum reads

$$
\mathcal{P}^{2} \equiv g_{\mu v} P^{\mu} P^{v}=-E^{2} / c^{2}+p^{2}=-m_{a} c^{2},
$$

with $E^{2} / c^{2}=-g_{00}\left(P^{0}\right)^{2}$ and $p^{2} \equiv g_{i j} P^{i} P^{j}$, and where $E$ is the neutrino energy and $p$ its physical (or proper) momentum. Because of the mass shell relation (13) without loss of generality we take only the spatial momentum components $P^{i}$ as independent variables [47]. The distribution function in the restricted phase space is then given by

$$
\left(P^{\mu} \frac{\partial}{\partial x^{\mu}}-\Gamma_{v \sigma}^{i} P^{v} P^{\sigma} \frac{\partial}{\partial P^{i}}\right) f\left(x^{\mu}, P^{i}\right)=0 .
$$

For a flat Friedmann-Robertson-Walker spacetime, the line element reads

$$
d s^{2}=-c^{2} d t^{2}+a^{2}(t)\left[d r^{2}+r^{2}\left(d \theta^{2}+\sin ^{2} d \phi^{2}\right)\right],
$$

where the time coordinate $(t)$ indicates the cosmic time and the spatial coordinates $(r, \theta, \phi)$ are comoving coordinates. For each $t$, the spatial slices are maximally symmetric, with $a(t)$ the scale factor that gauges how the distance between two points scales with time. The Christoffel symbols are given by $\Gamma_{00}^{0}=0, \Gamma_{0 i}^{0}=0, \Gamma_{i j}^{0}=\delta_{i j} a \dot{a} / c$, and $\Gamma_{0 j}^{i}=\delta_{j}^{i} H / c$, where $H=\dot{a} / a$ is the Hubble parameter.

From now on, we take $c=1$ to simplify notation. Substituting the coefficients of the affine connection into (14) we have

$$
\left(E \frac{\partial}{\partial t}+P^{i} \frac{\partial}{\partial x^{i}}-2 E H P^{i} \frac{\partial}{\partial P^{i}}\right) f\left(x^{\mu}, P^{i}\right)=0,
$$

where the factor of 2 reflects that the connection coefficients are symmetric in the lower indices.

Nevertheless, because of homogeneity of (FriedmannRobertson-Walker) spacetime, $f$ cannot depend on the spacial coordinates $x^{i}$, and so the second term in (16) is identically zero. Likewise, because of isotropy the phase space function can only depend on the absolute value of the momentum $P^{2}=\delta_{i j} P^{i} P^{j}$. This implies that 16 takes the form

$$
\left(\frac{\partial}{\partial t}-2 H P \frac{\partial}{\partial P}\right) f(t, P)=0
$$


or using the proper momentum can be rewritten as

$$
\left(\frac{\partial}{\partial t}-H p \frac{\partial}{\partial p}\right) f(t, p)=0,
$$

where we have made use of the fact that for a generic function $f=f\left(x^{2}\right)$ it follows that $x^{i}\left(\partial f / \partial x^{i}\right)=x(\partial f / \partial x)$, with $x^{2} \equiv \delta_{i j} x^{i} x^{j}$ [48].

Because of isotropy over the momentum space the neutrino number density relates to the phase space distribution according to

$$
n_{v}(t, p) d p=\frac{g}{(2 \pi)^{3}} 4 \pi p^{2} f(t, p) d p
$$

where we have allowed for $g=1 / 2$ internal degrees of freedom of Weyl spinors [49]. Multiplying (18) by $g d^{3} p /(2 \pi)^{3}$ it follows that

$$
\left(\frac{\partial}{\partial t}+3 H\right) n_{v}(t, p)=0,
$$

where the second term has been integrated by parts:

$$
-H \frac{g}{(2 \pi)^{3}} \int 4 \pi p^{3} d p \frac{\partial f}{\partial p}=3 H \frac{g}{(2 \pi)^{3}} \int 4 \pi p^{2} d p f .
$$

Even under the assumption that neutrinos propagate unscathed, the neutrino energy is redshifted by a factor of $(1+z)$ so we must correct (20) to account for the adiabatic energy losses $\left(E^{-1} d E / d t=H\right)$ and the source term. Introducing these two terms, 20) can be rewritten as

$$
\left(\frac{\partial}{\partial t}+3 H\right) n_{v_{\alpha}}(t, E)=\frac{\partial}{\partial E}\left[H E n_{v_{\alpha}}(E, t)\right]+Q_{s}(t, E) P_{\alpha s},
$$

where we have assumed that $m_{\mathrm{dc}} \gg m_{a}$ to adopt the ultra-relativistic approximation $E \approx p$, and where the source term $Q_{s}(t, E)$ describes the change of the net neutrino number density due to $\chi_{\mathrm{dc}} \rightarrow v_{s} \bar{v}_{s}$ decay [50].

\section{BUMP HUNTING}

We now turn to a comparison of the predictions of our scenario with the IceCube neutrino data. Following our previous study [18], we set $\tau_{\mathrm{dc}} \simeq 6 \times 10^{15} \mathrm{~s}$ and fix the fraction of $\chi_{\mathrm{dc}}$ particles to saturate the cosmological bound (3), yielding $F=4 \times 10^{-6}$. With the assumed 2body decay $\left(N_{v}=2\right)$ of $\chi_{\mathrm{dc}}$, the produced neutrino is monoenergetic, with energy $\varepsilon=m_{\mathrm{dc}} / 2$. The neutrino energy distribution from $\chi_{\mathrm{dc}}$ decay is given by $d N_{v} / d E=$ $N_{v} \delta(E-\varepsilon)$.

The source term takes the form

$$
Q_{s}(t, E)=\frac{n_{\mathrm{dc}}(t)}{\tau_{\mathrm{dc}}} \frac{d N_{v}}{d E},
$$

where

$$
n_{\mathrm{dc}}(t)=Y_{\mathrm{dc}} s(t) e^{-t / \tau_{\mathrm{dc}}}
$$

is the number density of $\chi_{\mathrm{dc}}, s(t)$ is the entropy density with $s\left(t_{0}\right) \simeq 2.9 \times 10^{3} \mathrm{~cm}^{-3}$, and

$$
Y_{\mathrm{dc}}=3.6 \times 10^{-9} \frac{F \Omega_{\mathrm{dm}} h^{2}}{m_{\mathrm{dc}} / \mathrm{GeV}}
$$

is the comoving number density at the CMB epoch.

We solve (22) using the Green's function method, with $G_{\alpha s}\left(t^{\prime}, E^{\prime} ; t, E\right)$ satisfying

$$
\left(\frac{\partial}{\partial t}+2 H-H E \frac{\partial}{\partial E}\right) G_{\alpha s}\left(t^{\prime}, E^{\prime} ; t, E\right)=\delta_{s \alpha} \delta\left(t^{\prime}-t\right) \delta\left(E^{\prime}-E\right) \quad \text { and } \quad G_{s \alpha}\left(t^{\prime}, E^{\prime} ; t, E\right)_{t^{\prime}>t}=0
$$

Then,

$$
G_{\alpha s}\left(t^{\prime}, E^{\prime} ; t, E\right)=\Theta\left(t-t^{\prime}\right)\left\{\mathcal{K} e^{-\int_{t}^{t^{\prime}}(-2 H) d t^{\prime \prime}}+\int e^{-\int_{t}^{t^{\prime}}(-2 H) d t^{\prime \prime}} d t^{\prime} \delta_{s \alpha} \delta\left(E^{\prime}-a(t) E / a\left(t^{\prime}\right)\right)\right\},
$$

where $a(t)$ is the scale factor at cosmic time $t$ and $\mathcal{K}$ the integration constant [16]. The integral in the exponential is given by

$$
\int_{t}^{t^{\prime}} 2 H d t^{\prime \prime}=\int_{t}^{t^{\prime}} 2 \frac{d a / d t^{\prime \prime}}{a} d t^{\prime \prime}=\ln \left(\frac{a\left(t^{\prime}\right)}{a(t)}\right)^{2} .
$$

Now, without loss of generality we set $\mathcal{K}=0$ to obtain

$$
G_{s \alpha}\left(t^{\prime}, E^{\prime} ; t, E\right)=\Theta\left(t-t^{\prime}\right) \int\left(\frac{a\left(t^{\prime}\right)}{a(t)}\right)^{2} d t^{\prime} \delta_{\alpha s} \delta\left(E^{\prime}-a(t) E / a\left(t^{\prime}\right)\right) .
$$

The density of neutrinos of flavor $\alpha$ at IceCube is found to be

$$
n_{v_{\alpha}}\left(t_{0}, E\right)=\int_{t_{0}}^{\mathcal{T}} d t^{\prime} \int d E^{\prime} Q_{s}\left(t^{\prime}, E^{\prime}\right) P_{s \beta} G_{\beta \alpha}\left(t^{\prime}, E^{\prime} ; t, E\right),
$$


where

$$
\mathcal{T}=\frac{1}{H_{0}} \int_{0}^{[1+z(\mathcal{T})]^{-1}} \frac{a d a}{\sqrt{\Omega_{\Lambda} a^{4}+\Omega_{m} a+\Omega_{r}}} \simeq \frac{2}{3 H_{0} \sqrt{\Omega_{\Lambda}}} \sinh ^{-1}\left[\sqrt{\frac{\Omega_{\Lambda}}{\Omega_{m}}}\left(\frac{E}{\varepsilon}\right)^{3 / 2}\right] \simeq A\left(\frac{E}{\varepsilon}\right)^{3 / 2}
$$

In the solution to the integral in (31), we neglect decays prior to recombination so we can omit $\Omega_{r}$. We use $a_{\max }=$ $[1+z(\mathcal{T})]^{-1}=E / \varepsilon$. Given $h=0.6766, \Omega_{\Lambda}=0.6889$ and $\Omega_{m}=0.3111$ [28], the constant $A$ is

$$
A=\frac{2}{3 H_{0} \sqrt{\Omega_{m}}}=5.45 \times 10^{17} \mathrm{~s} .
$$

Note that $H(\mathcal{T}) \simeq H_{0} \sqrt{\Omega_{m}}[1+z(\mathcal{T})]^{3 / 2}$ when matter dominates, so $[H(\mathcal{T})]^{-1} \simeq 8.17 \times 10^{17}(E / \varepsilon)^{3 / 2} \mathrm{~s}$ for $[1+z(\mathcal{T})] \gg$ 1.30 in the matter dominated era. by

The density of neutrinos of flavor $\alpha$ at Earth is given

$$
n_{v_{\alpha}}\left(t_{0}, E\right)=\frac{N_{v} Y_{\mathrm{dc}} s\left(t_{0}\right)}{\tau_{\mathrm{dc}} E} \frac{e^{-\mathcal{T} / \tau_{\mathrm{dc}}}}{H(\mathcal{T})} P_{s \alpha}
$$

Finally, the all-flavor flux of active SM neutrinos at IceCube is found to be

$$
\begin{aligned}
E^{2} \Phi_{(v+\bar{v}) s \mathrm{~s}}\left(t_{0}, E\right) & =\sum_{e, \mu, \tau} \frac{c}{4 \pi} E^{2} n_{v_{\alpha}}\left(t_{0}, E\right) \\
& =\frac{c}{4 \pi} \frac{N_{v} Y_{\mathrm{dc}} s\left(t_{0}\right)}{\tau_{\mathrm{dc}}} \frac{E e^{-\mathcal{T} / \tau_{\mathrm{dc}}}}{H(\mathcal{T})} \varkappa,
\end{aligned}
$$

where

$$
\varkappa=\sum_{\alpha=e, \mu, \tau} P_{\alpha s}=\sum_{\alpha, a}\left|U_{\alpha a}\right|^{2}\left|U_{s a}\right|^{2} .
$$

Equation (34) can be written approximately as

$$
E^{2} \Phi_{(v+\bar{v}) \mathrm{SM}} \simeq \mathcal{N}\left(F \Gamma_{\mathrm{dc}} \mathcal{\varkappa}\right)\left(\frac{E}{\varepsilon}\right)^{5 / 2} \exp \left[-\frac{A}{\tau_{\mathrm{dc}}}\left(\frac{E}{\varepsilon}\right)^{3 / 2}\right]
$$

where

$$
\mathcal{N}=2.43 \times 10^{21} \frac{N_{v}}{2} \frac{\mathrm{GeV}}{\mathrm{cm}^{2} \mathrm{sr}},
$$

given $\Omega_{\mathrm{dm}} h^{2}=0.1193$ [28]. The maximum of the distribution of 36 occurs at

$$
E_{\text {peak }} \simeq \frac{m_{\mathrm{dc}}}{2}\left(\frac{5 \tau_{\mathrm{dc}}}{3 A}\right)^{2 / 3}=\frac{m_{\mathrm{dc}}}{2}\left(\frac{\tau_{\mathrm{dc}}}{3.27 \times 10^{17} \mathrm{~s}}\right)^{2 / 3}
$$

The IceCube data fix the location of the peak of $E^{2} \Phi_{(v+\bar{v})_{S M}}$ [3]. A range of mass and lifetime combinations yield a peak in the energy bin with the highest $E^{2} \Phi_{(v+\bar{v})_{S M}}$, namely, for $8.36 \times 10^{4} \leq E / \mathrm{GeV} \leq 1.86 \times 10^{5}$, an average of $1.37 \times 10^{5} \mathrm{GeV}$.

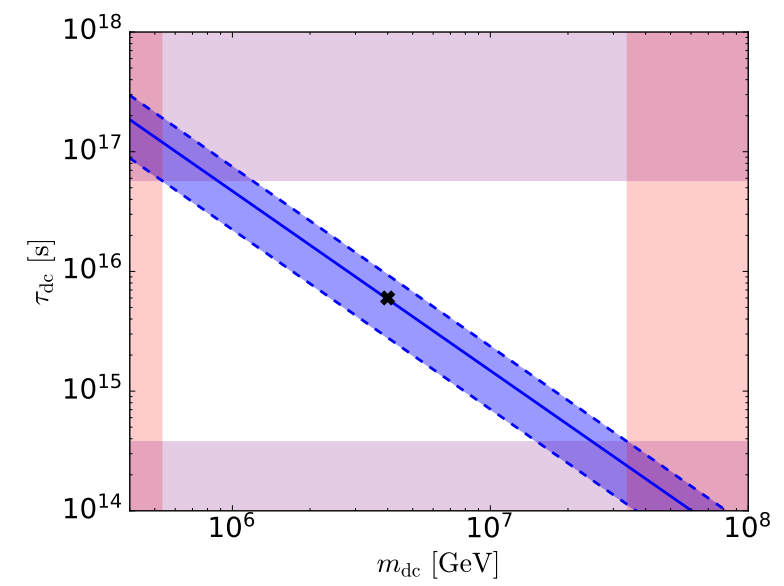

FIG. 1: The lifetime $\tau_{\mathrm{dc}}$ versus $m_{\mathrm{dc}}$ for $E_{\text {peak }}=1.37 \times 10^{5} \mathrm{GeV}$ (solid line), $8.36 \times 10^{4} \mathrm{GeV}$ (lower dashed line) and $1.87 \times 10^{5}$ $\mathrm{GeV}$ (upper dashed line). The shaded purple regions are excluded based on the requirement that $\chi_{\mathrm{dc}} \rightarrow v_{s} \bar{v}_{s}$ decays contribute less than $1 \%$ of the peak of $E^{2} \Phi_{(v+\bar{v})_{S M}}$, so the shaded red regions correspond to excluded values of $m_{\mathrm{dc}}$, accounting for the range of $E_{\text {peak }}$. The cross marks the fiducial point of our analysis.

For $E_{\text {peak }}=1.37 \times 10^{5} \mathrm{GeV}$ and $\tau_{\mathrm{dc}}=6 \times 10^{15} \mathrm{~s}, m_{\mathrm{dc}}=$ $4 \times 10^{6} \mathrm{GeV}$. Note, $\varepsilon / E_{\text {peak }}=\left(1+z_{\text {peak }}\right)=14.6$ for this value of $m_{\mathrm{dc}}$. For larger masses, the lifetime is shorter, for example, $\tau_{\mathrm{dc}}=3.8 \times 10^{14} \mathrm{~s}$ for $m_{\mathrm{dc}}=2.5 \times 10^{7} \mathrm{GeV}$. For lighter masses, the lifetimes approach the age of the universe, for example, $m_{\mathrm{dc}}=10^{6} \mathrm{GeV}$ corresponds to $\tau_{\mathrm{dc}}=4.7 \times 10^{16} \mathrm{~s}$. Figure 1 shows $\tau_{\mathrm{dc}}$ as a function of $m_{\mathrm{dc}}$ for $E_{\text {peak }}=1.37 \times 10^{5} \mathrm{GeV}$ (solid line) and for $E_{\text {peak }}=$ $8.36 \times 10^{4}-1.87 \times 10^{5} \mathrm{GeV}$ (shaded blue band). The cross marks the location of the fiducial values $\tau_{\mathrm{dc}}=6 \times 10^{15} \mathrm{~s}$ and $m_{\mathrm{dc}}=4 \times 10^{6} \mathrm{GeV}$

The flux of SM neutrinos from $\chi_{\mathrm{dc}} \rightarrow v_{s} \bar{v}_{s}$, scaled by $E^{2}$, as a function of $E / \varepsilon$ is shown in Fig. 2 Because of the form of (36), the shape is the same for any $\varepsilon=$ $m_{\mathrm{dc}} / 2$ and lifetime, shifted in $E / \varepsilon$ because of $m_{\mathrm{dc}}$. In Fig. 2. the flux has been normalized so that the peak of the $\chi_{\mathrm{dc}} \rightarrow v_{s} \bar{v}_{s}$ contribution is $E^{2} \Phi_{(v+\bar{v})_{S M}}=5.80 \times 10^{-8}$ $\mathrm{GeV} /\left(\mathrm{cm}^{2} \mathrm{ssr}\right)$. The lifetime is chosen from with $E_{\text {peak }}=1.37 \times 10^{5} \mathrm{GeV}$.

As Figs. 1 and 2 show, a larger $m_{\mathrm{dc}}$ corresponds to a shorter lifetime and to the correspondingly larger redshifts to the neutrino energy $\varepsilon$ at production. If the lifetime is too short, $\chi_{\mathrm{dc}}$ decays will occur before recombination. The requirement that the $E^{2} \Phi_{(v+\bar{v})_{s M}}$ be $<1 \%$ of the peak value from $\chi_{\mathrm{dc}}$ decays at $z=1100$ corresponds to $\tau_{\mathrm{dc}}>3.8 \times 10^{14} \mathrm{~s}$. 


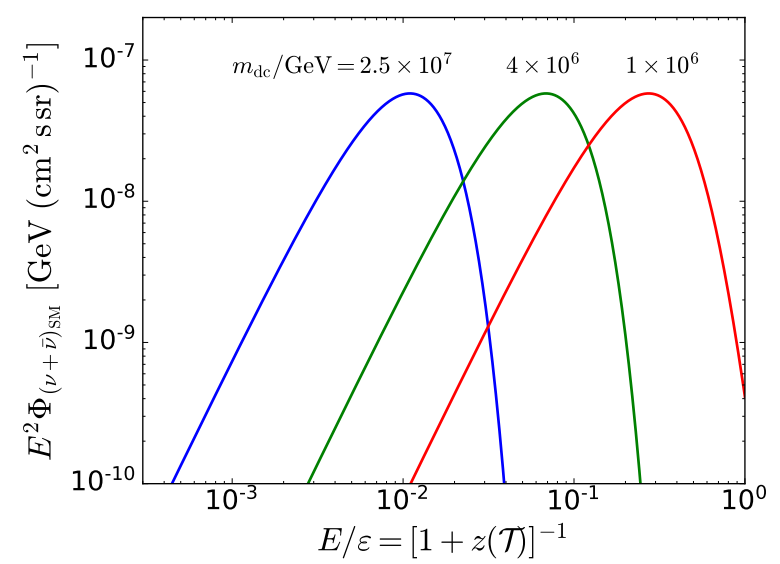

FIG. 2: The quantity $E^{2} \Phi_{(v+\bar{v})_{S M}}$ as a function of $E / \varepsilon$ assuming $E_{\text {peak }}=1.37 \times 10^{5} \mathrm{GeV}$ and the decay of $\chi_{\mathrm{dc}}$ to sterile neutrinos contributes $\max \left(E^{2} \Phi_{(v+\bar{v})_{S M}}\right)=5.80 \times 10^{-8} \mathrm{GeV} /\left(\mathrm{cm}^{2} \mathrm{~s} \mathrm{sr}\right)$.

For smaller masses, we find an upper bound on the lifetime of $\tau_{\mathrm{dc}}^{\max }=5.8 \times 10^{16} \mathrm{~s}$ if we require that for $z=0$, $E^{2} \Phi_{(v+\bar{v})_{S M}}$ be $<1 \%$ of the peak value from $\chi_{\mathrm{dc}}$ decays. The two exclusion regions are shown by shaded purple bands in Fig. 11 The shaded red bands correspond to excluded $m_{\mathrm{dc}}$ values, based on (38), accounting for the bin width of $E_{\text {peak }}$ that corresponds to the blue band. In what follows, we assume $E_{\text {peak }}=1.37 \times 10^{5} \mathrm{GeV}$.

For the $\chi_{\mathrm{dc}}$ decay width in terms of an effective coupling $g_{\text {eff }}, \Gamma_{\mathrm{dc}}=g_{\text {eff }}^{2} m_{\mathrm{dc}} /(16 \pi)$, the lifetimes and masses considered here require $g_{\text {eff }} \sim 5 \times 10^{-23}$. Given $\tau_{\mathrm{dc}}^{\max }=$ $5.8 \times 10^{16} \mathrm{~s}$, the bound on $F \Gamma_{\mathrm{dc}}$ in 3 translates to

$$
F<4.14 \times 10^{-5},
$$

several orders of magnitude lower than from the more general constraint on $F$ from $\mathrm{CMB}$ measurements.

With the approximate formulas for $\mathcal{T}$ and $H(\mathcal{T})$ and the relation between $m_{\mathrm{dc}}$ and $\tau_{\mathrm{dc}}$, one can show that the quantity $F \varkappa$ scales with $m_{\mathrm{dc}}$ as

$$
F \varkappa \simeq 6.0 \times 10^{-10}\left(\frac{m_{\mathrm{dc}}}{4 \times 10^{6} \mathrm{GeV}}\right) .
$$

For the mass range discussed here, $8.7 \times 10^{5} \leq m_{\mathrm{dc}} / \mathrm{GeV} \leq$ $2.5 \times 10^{7}$, we find $1.3 \times 10^{-10} \leq F \varkappa \leq 3.8 \times 10^{-9}$. Given (39), $\varkappa>3.2 \times 10^{-6}$. For our fiducial values, $m_{\mathrm{dc}}=4 \times 10^{6} \mathrm{GeV}$, $\tau_{\mathrm{dc}}=6 \times 10^{15} \mathrm{~s}$, and $F=4 \times 10^{-6}$ we obtain $Y_{\mathrm{dc}} \simeq 4.3 \times 10^{-22}$ and $\varkappa=1.5 \times 10^{-4}$. This value of $\kappa$ can be achieved in a scenario with $\left|U_{s 4}\right|^{2} \simeq 1$ and $\left|U_{\alpha 4}\right|^{2} \lesssim 1.5 \times 10^{-4}$ for $\alpha=e, \mu, \tau$, values well below experimental constraints on active-sterile mixing [51] and much smaller than $\left|U_{e 3}\right|^{2} \simeq$ $2 \times 10^{-2}$. For $\varkappa \sim 10^{-4}$ and $m_{v_{s}} \sim 1 \mathrm{eV}$, active-sterile oscillations in cosmology will not violate bounds on the effective number of neutrino species, as discussed in, e.g., Ref. [52].

In Fig. 3 we show the $\gamma$-ray flux and the monoenergetic all-flavor flux of active SM neutrinos, smeared by

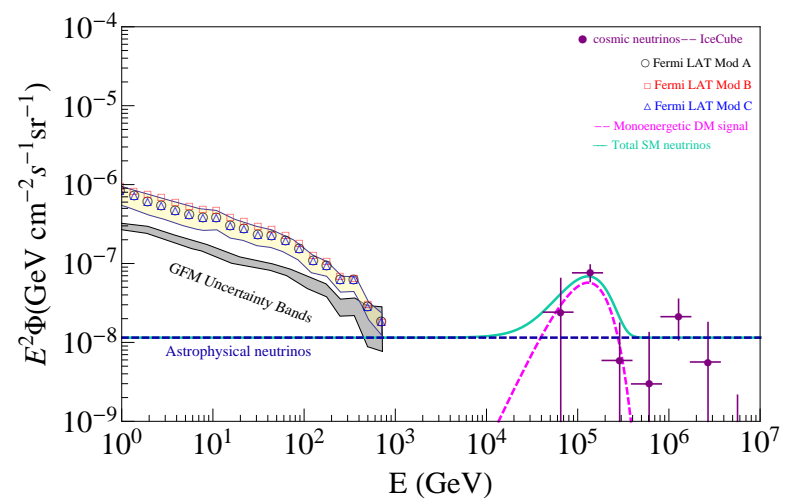

FIG. 3: The total extragalactic $\gamma$-ray background (open symbols) reported by the Fermi Collaboration [6] and the diffuse astrophysical flux (solid symbols) reported by the IceCube Collaboration (frequentist statistcal analysis $7.5 \mathrm{yr}$ data) [3]. The $\gamma$-ray result is shown for three foreground models, with the yellow band showing background modeling uncertainties and the gray band showing the cumulative intensity from resolved Fermi sources at latitudes $|b|>20^{\circ}$. The SM diffuse astrophysical neutrino flux is shown with the horizontal dashed line, and the SM neutrino flux from dark matter decays is shown with the magenta dashed line.

redshift evolution, together with the diffuse astrophysical neutrino flux superimposed on IceCube data. The horizontal line in Fig. 3indicates the flux of astrophysical neutrinos produced via pion decay, which translates to equal fluxes of neutrino flavors and is taken to saturate the Fermi flux, given by

$$
\frac{\Phi_{(v+\bar{v})_{\mathrm{SM}, \text { astro }}}(E)}{\mathrm{GeV} \mathrm{cm}^{2} \mathrm{sr} \mathrm{s}}=1.5 \times 10^{-18}\left(\frac{E}{10^{5} \mathrm{GeV}}\right)^{-2} .
$$

A cutoff (or steepening) of the astrophysical neutrino spectrum around $E \sim 10^{7} \mathrm{GeV}$ is necessary to accommodate the non-observation of $v_{e}$ or $v_{\tau}$ events above this energy [53]. The astrophysical neutrino flux adopted herein is consistent at the $1 \sigma$ level with the muon neutrino flux reported by the IceCube Collaboration [54]. The (magenta) dashed line is obtained for our fiducial parameters, or alternatively, with a combination of parameters that satisfy (38) and (40). The active flavor fractions of this all-flavor neutrino flux at Earth are essentially unconstrained, as discussed in [44].

\section{RELATED PHENOMENOLOGY}

Sterile neutrinos relevant to the MiniBooNE [55] and LSND [56] anomalies have masses $1 \lesssim m_{s} / \mathrm{eV} \lesssim 10$ and active-sterile neutrino mixing with an amplitude at the level of 0.01 . IceCube places constraints on this $3+1$ oscillation framework by searching for Earth matter effects in oscillations of atmospheric neutrinos with energies between $200 \mathrm{GeV}$ and $10 \mathrm{TeV}$ [57]. However, for the $100 \mathrm{TeV}$ energies of our scenario, no such matter effects 
are observable at IceCube. This conclusion also applies for sterile neutrino masses above $10 \mathrm{eV}$ [58].

Proposed neutrino detection experiments along the LHC beamline such as FASER [59-61] and FASER $[$ [62, 63], XsEN [64, 65] and SNDLHC [66] have the potential to probe active-sterile mixing. Neutrinos that travel down the beamline are produced by forward pions and kaons that decay outside the detector, from $W$ and $Z$ decays, and from prompt decays of charmed hadrons. Indeed, in the far forward region, the dominant source tau neutrinos is from $D_{s}^{ \pm}$decays [67], making $v_{\tau} \rightarrow v_{s}$ oscillations the cleanest signal. Even so, large uncertainties in forward charm production kinematic distributions and cross sections mean that until charm production calculations are better refined with new forward production data, signals of $v_{\tau} \rightarrow v_{s}$ oscillations would be via observations of spectral distortions [68]. With baselines of these far-forward experiments on the scale of $500 \mathrm{~m}$, a characteristic sterile neutrino mass scale of 10 's of eV will show oscillation dips for neutrino energies of order $\sim 150$ $\mathrm{GeV}$ if mixing angles are large enough. To be observable, sufficiently large values of $\varkappa$ are required, in particular, that $\left|U_{\tau 4}\right|^{2} \simeq 0.15$, close to the maximum allowed value [51]. To avoid the cosmological constraints on such a large mixing angle, additional "secret interactions" (viz. $C[f] \neq 0$ ) could be invoked, as in, e.g., Refs. [69-71].

\section{CONCLUSIONS}

We propose a novel explanation of the spectrum of cosmic neutrinos on the basis of cold dark matter which decays into sterile neutrinos that, after oscillations, produce the bumpy signal observed in IceCube data. The dark matter particle decays via $\chi_{\mathrm{dc}} \rightarrow v_{s} \bar{v}_{s}$ with a branching fraction of essentially unity, and thereby eliminates the IceCube-Fermi tension. The scenario features interesting phenomenology which can be summarized as follows:

- The total decay width of the dark matter particle satisfies $H_{0}<\Gamma_{\mathrm{dc}} \lesssim H\left(z_{\mathrm{LS}}\right)$. The lower limit ensures that most of the $\chi_{\mathrm{dc}}$ particles have disappeared by $z=3$, so monoenergetic neutrinos are not found in IceCube searches of dark matter decay in the Galactic center [72]. The upper limit on $\Gamma_{\mathrm{dc}}$ ensures that the portion of the overall dark matter abundance that has been depleted by decays prior to last scattering is negligible, avoiding modifications of $\Lambda C D M$ predictions from big bang nucleosynthesis or $\mathrm{CMB}$ phenomenology.

- A subdominant CDM component decaying into dark radiation after recombination depletes dark matter density at low redshifts reducing the power of the CMB lensing effect, which is at odds with Planck data. This sets an upper limit of $F \Gamma_{\mathrm{dc}}<$ $2.25 \times 10^{-5} \mathrm{Gyr}^{-1}$ at $95 \%$ CL [30]. Normalization to the IceCube flux yields a constraint on $\kappa F \Gamma_{\mathrm{dc}}$ after sterile-active neutrino oscillations, with mixing amplitude $\propto \kappa$.

- The thermalization of extra light species in the early universe modifies the energy density of radiation. The presence of additional relativistic degrees of freedom can be characterized by the number of equivalent light neutrino species $N_{\text {eff }} \equiv$ $\left(\rho_{\mathbb{R}}-\rho_{\gamma}\right) / \rho_{v}$ in units of the density of a single Weyl neutrino $\rho_{v}$, where $\rho_{\mathrm{R}}$ is the total energy density in relativistic particles and $\rho_{\gamma}$ is the energy density of photons [73]. For $1 \lesssim m_{s} / \mathrm{eV} \lesssim 10$ and our fiducial value $\varkappa=1.5 \times 10^{-4}$, the mixing is not large enough to allow a full energy transfer between the sterile and the active states [52]. Indeed, due to incomplete thermalization the model prediction for $N_{\text {eff }}$ is virtually indistinguishable from that of three families of massless SM neutrinos $\left(N_{\text {eff }}=3.046\right.$ [74] $)$ in $\Lambda \mathrm{CDM}$ cosmology.

- The allowed range for the sterile neutrino mass encompasses the mass scale of the long-standing anomalies in MiniBooNE [55] and LSND [56]. However, for the preferred region of active-sterile mixing parameters from short-baseline neutrino experiments, the sterile neutrino is fully thermalized $\left(N_{\text {eff }} \simeq 4\right)$ and therefore in strong tension with the upper limit derived by the Planck Collaboration: $N_{\text {eff }}=3.12_{-0.26}^{+0.25}$ at the $95 \%$ CL [28].

- The energy of the sterile neutrino flux is outside the range of oscillation searches for sterile neutrinos at IceCube [57].

- Proposed neutrino detection experiments along the LHC beamline will be sensitive to active-sterile neutrino mixing if $\kappa$ is sufficiently large [68]. To avoid the cosmological constraints on such a large mixing with the hidden sector, a collision term $(C[f] \neq 0)$ should be added to $(10)$ to account for secret neutrino interactions [69-71].

In summary, we have proposed a new scenario to explain IceCube data. This scenario can be confronted with future data from IceCube-Gen2 [75] and KM3NeT [76]. Observation by both of these neutrino-detection facilities will be able to test whether the bumpy signal has a cosmological origin.

\section{Acknowledgments}

L.A.A. is supported by the U.S. National Science Foundation (NSF) Grant PHY-1620661 and the National Aeronautics and Space Administration (NASA) Grant 80NSSC18K0464. V.B. is supported by the U.S. Department of Energy (DoE) Grant DE-SC-0017647. D.M. is supported by the DoE Grant DE-SC-0010504. M.H.R. is supported by the DoE Grant DE-SC-0010113. T.J.W. is supported by the DoE Grant DE-SC-0011981. T.J.W. thanks Vanderbilt University and its Physics Office for naming him Emeritus Professor of Physics, effective upon his retirement December 31, 2020. Any opinions, findings, and conclusions or recommendations ex- 
pressed in this material are those of the authors and do not necessarily reflect the views of the NSF, NASA, or DoE.

\section{Note added}

After our paper was published, the authors of Ref. [30] corrected the 95\% CL upper bounds in Eqs. (2) and (3) to

$$
F<2.62 \times 10^{-2}
$$

and

$$
F \Gamma_{\mathrm{dc}}<5.84 \times 10^{-2} \mathrm{Gyr}^{-1}
$$

Since our fiducial values are comfortably consistent with the revised constraints, our conclusions remain unchanged.
[1] M. G. Aartsen et al. [IceCube Collaboration], Evidence for high-energy extraterrestrial neutrinos at the IceCube detector, Science 342, 1242856 (2013) doi:10.1126/science.1242856 arXiv:1311.5238 [astroph.HE]].

[2] M. G. Aartsen et al. [IceCube Collaboration], Observation of high-energy astrophysical neutrinos in three years of IceCube data, Phys. Rev. Lett. 113, 101101 (2014) doi:10.1103/PhysRevLett.113.101101 arXiv:1405.5303 [astro-ph.HE]].

[3] R. Abbasi et al. [IceCube Collaboration], The IceCube high-energy starting event sample: Description and flux characterization with 7.5 years of data, |arXiv:2011.03545 [astro-ph.HE]].

[4] E. Waxman and J. N. Bahcall, High-energy neutrinos from astrophysical sources: An upper bound, Phys. Rev. D 59, 023002 (1999) doi:10.1103/PhysRevD.59.023002 |arXiv:hep-ph/9807282 [hep-ph]].

[5] J. Alvarez-Muniz and F. Halzen, Possible high-energy neutrinos from the cosmic accelerator RX J1713.7-3946, Astrophys. J. Lett. 576, L33-L36 (2002) doi:10.1086/342978 arXiv:astro-ph/0205408 [astro-ph]].

[6] M. Ackermann et al. [Fermi-LAT Collaboration], The spectrum of isotropic diffuse gamma-ray emission between $100 \mathrm{MeV}$ and $820 \mathrm{GeV}$, Astrophys. J. 799, 86 (2015) doi:10.1088/0004-637X/799/1/86 |arXiv:1410.3696 [astroph.HE]].

[7] K. Murase, D. Guetta and M. Ahlers, Hidden cosmicray accelerators as an origin of $\mathrm{TeV}-\mathrm{PeV}$ cosmic neutrinos, Phys. Rev. Lett. 116, no.7, 071101 (2016) doi:10.1103/PhysRevLett.116.071101 arXiv:1509.00805 [astro-ph.HE]].

[8] A. Capanema, A. Esmaili and K. Murase, New constraints on the origin of medium-energy neutrinos observed by IceCube, Phys. Rev. D 101, no.10, 103012 (2020) doi:10.1103/PhysRevD.101.103012 |arXiv:2002.07192 [hep-ph]].

[9] A. Capanema, A. Esmaili and P. D. Serpico, Where do IceCube neutrinos come from? Hints from the diffuse gamma-ray flux, |arXiv:2007.07911[hep-ph]].

[10] M. Ahlers, L. A. Anchordoqui, H. Goldberg, F. Halzen, A. Ringwald and T. J. Weiler, Neutrinos as a diagnostic of cosmic ray galactic/extra-galactic transition, Phys. Rev. D 72, 023001 (2005) doi:10.1103/PhysRevD.72.023001 arXiv:astro-ph/0503229 [astro-ph]].

[11] B. Feldstein, A. Kusenko, S. Matsumoto and T. T. Yanagida, Neutrinos at IceCube from heavy decaying dark matter, Phys. Rev. D 88, no.1, 015004 (2013) doi:10.1103/PhysRevD.88.015004 |arXiv:1303.7320 [hep-ph]].

[12] A. Esmaili and P. D. Serpico, Are IceCube neutrinos unveiling PeV-scale decaying dark matter?, JCAP 11, 054 (2013) doi:10.1088/1475-7516/2013/11/054 |arXiv:1308.1105 [hep-ph]].

[13] Y. Bai, R. Lu and J. Salvado, Geometric compatibility of IceCube TeV-PeV neutrino excess and its Galactic dark matter origin, JHEP 01, 161 (2016) doi:10.1007/JHEP01(2016)161 arXiv:1311.5864[hep-ph]].

[14] Y. Ema, R. Jinno and T. Moroi, Cosmic-ray neutrinos from the decay of long-lived particle and the recent IceCube result, Phys. Lett. B 733, 120-125 (2014) doi:10.1016/j.physletb.2014.04.021 |arXiv:1312.3501 [hepph]].

[15] A. Bhattacharya, M. H. Reno and I. Sarcevic, Reconciling neutrino flux from heavy dark matter decay and recent events at IceCube, JHEP 06, 110 (2014) doi:10.1007/JHEP06(2014)110 |arXiv:1403.1862 [hep-ph]].

[16] Y. Ema, R. Jinno and T. Moroi, Cosmological implications of high-energy neutrino emission from the decay of long-lived particle, JHEP 10, 150 (2014) doi:10.1007/JHEP10(2014)150 |arXiv:1408.1745 [hep-ph]].

[17] C. Rott, K. Kohri and S. C. Park, Superheavy dark matter and IceCube neutrino signals: Bounds on decaying dark matter, Phys. Rev. D 92, no.2, 023529 (2015) doi:10.1103/PhysRevD.92.023529 |arXiv:1408.4575 [hep$\mathrm{ph}]$.

[18] L. A. Anchordoqui, V. Barger, H. Goldberg, X. Huang, D. Marfatia, L. H. M. da Silva and T. J. Weiler, IceCube neutrinos, decaying dark matter, and the Hubble constant, Phys. Rev. D 92, no.6, 061301 (2015) [erratum: Phys. Rev. D 94, no.6, 069901 (2016)] doi:10.1103/PhysRevD.94.069901 |arXiv:1506.08788 [hep-ph]].

[19] A. Bhattacharya, A. Esmaili, S. Palomares-Ruiz and I. Sarcevic, Probing decaying heavy dark matter with the 4-year IceCube HESE data, JCAP 07, 027 (2017) doi:10.1088/1475-7516/2017/07/027 |arXiv:1706.05746 [hep-ph]].

[20] M. Chianese, G. Miele and S. Morisi, Interpreting IceCube 6-year HESE data as an evidence for hundred TeV decaying Dark Matter, Phys. Lett. B 773, 591-595 (2017) doi:10.1016/j.physletb.2017.09.016 arXiv:1707.05241[hep$\mathrm{ph}]$.

[21] A. Bhattacharya, A. Esmaili, S. Palomares-Ruiz and I. Sarcevic, Update on decaying and annihilating heavy 
dark matter with the 6-year IceCube HESE data, JCAP 05, 051 (2019) doi:10.1088/1475-7516/2019/05/051 |arXiv:1903.12623 [hep-ph]].

[22] K. Murase, R. Laha, S. Ando and M. Ahlers, Testing the dark matter scenario for $\mathrm{PeV}$ neutrinos observed in IceCube, Phys. Rev. Lett. 115, no.7, 071301 (2015) doi:10.1103/PhysRevLett.115.071301 |arXiv:1503.04663 [hep-ph]].

[23] M. Ahlers, Y. Bai, V. Barger and R. Lu, Galactic neutrinos in the TeV to PeV range, Phys. Rev. D 93, no.1, 013009 (2016) doi:10.1103/PhysRevD.93.013009 |arXiv:1505.03156 [hep-ph]].

[24] A. Esmaili and P. D. Serpico, Gamma-ray bounds from EAS detectors and heavy decaying dark matter constraints, JCAP 10, 014 (2015) doi:10.1088/14757516/2015/10/014 |arXiv:1505.06486 [hep-ph]].

[25] Z. Berezhiani, Shadow dark matter, sterile neutrinos and neutrino events at IceCube, Nucl. Part. Phys. Proc. 265266, 303-306 (2015) doi:10.1016/j.nuclphysbps.2015.06.076 |arXiv:1506.09040 [astro-ph.HE]].

[26] G. Bertone, D. Hooper and J. Silk, Particle dark matter: Evidence, candidates and constraints, Phys. Rept. 405, 279390 (2005) doi:10.1016/j.physrep.2004.08.031 |arXiv:hepph/0404175 [hep-ph]].

[27] K. R. Dienes and B. Thomas, Dynamical dark matter I: theoretical overview, Phys. Rev. D 85, 083523 (2012) doi:10.1103/PhysRevD.85.083523 arXiv:1106.4546 [hep$\mathrm{ph}]$.

[28] N. Aghanim et al. [Planck Collaboration], Planck 2018 results. VI. Cosmological parameters, Astron. Astrophys. 641, A6 (2020) doi:10.1051/0004-6361/201833910 |arXiv:1807.06209 [astro-ph.CO]].

[29] K. S. Dawson et al. [BOSS Collaboration], The Baryon Oscillation Spectroscopic Survey of SDSS-III, Astron. J. 145, 10 (2013) doi:10.1088/0004-6256/145/1/10 |arXiv:1208.0022 [astro-ph.CO]].

[30] A. Nygaard, T. Tram and S. Hannestad, Updated constraints on decaying cold dark matter, |arXiv:2011.01632 [astro-ph.CO]].

[31] Z. Berezhiani, A. D. Dolgov and I. I. Tkachev, Reconciling Planck results with low redshift astronomical measurements, Phys. Rev. D 92, no.6, 061303 (2015) doi:10.1103/PhysRevD.92.061303 |arXiv:1505.03644 [astro-ph.CO]].

[32] A. Chudaykin, D. Gorbunov and I. Tkachev, Dark matter component decaying after recombination: Lensing constraints with Planck data, Phys. Rev. D 94, 023528 (2016) doi:10.1103/PhysRevD.94.023528 |arXiv:1602.08121|.

[33] V. Poulin, P. D. Serpico and J. Lesgourgues, A fresh look at linear cosmological constraints on a decaying dark matter component, JCAP 08, 036 (2016) doi:10.1088/14757516/2016/08/036 |arXiv:1606.02073 [astro-ph.CO]].

[34] A. Chudaykin, D. Gorbunov and I. Tkachev, Dark matter component decaying after recombination: Sensitivity to baryon acoustic oscillation and redshift space distortion probes, Phys. Rev. D 97, no.8, 083508 (2018) doi:10.1103/PhysRevD.97.083508 |arXiv:1711.06738 [astro-ph.CO]].

[35] K. Vattis, S. M. Koushiappas and A. Loeb, Dark matter decaying in the late Universe can relieve the $H_{0}$ tension, Phys. Rev. D 99, no.12, 121302 (2019) doi:10.1103/PhysRevD.99.121302 |arXiv:1903.06220 [astro-ph.CO]].

[36] S. J. Clark, K. Vattis and S. M. Koushiappas, CMB con- straints on late-universe decaying dark matter as a solution to the $H_{0}$ tension, [arXiv:2006.03678 [astro-ph.CO]].

[37] G. F. Abellan, R. Murgia, V. Poulin and J. Lavalle, Hints for decaying dark matter from $S_{8}$ measurements, [arXiv:2008.09615 [astro-ph.CO]].

[38] L. A. Anchordoqui, Decaying dark matter, the $H_{0}$ tension, and the lithium problem, Phys. Rev. D (to be published) |arXiv:2010.09715 [hep-ph]].

[39] T. J. Weiler, Resonant absorption of cosmic ray neutrinos by the relic neutrino background, Phys. Rev. Lett. 49, 234 (1982) doi:10.1103/PhysRevLett.49.234

[40] M. C. Gonzalez-Garcia and M. Maltoni, Phenomenology with massive neutrinos, Phys. Rept. 460, 1-129 (2008) doi:10.1016/j.physrep.2007.12.004 |arXiv:0704.1800 [hepph]].

[41] V. D. Barger, S. Pakvasa, T. J. Weiler and K. Whisnant, Variations on four neutrino oscillations, Phys. Rev. D 58, 093016 (1998) doi:10.1103/PhysRevD.58.093016 [arXiv:hep-ph/9806328 [hep-ph]].

[42] S. I. Dutta, M. H. Reno and I. Sarcevic, High-energy neutrino signals of four neutrino mixing, Phys. Rev. D 64, 113015 (2001) doi:10.1103/PhysRevD.64.113015 [arXiv:hep-ph/0104275 [hep-ph]].

[43] C. A. Argüelles, K. Farrag, T. Katori, R. Khandelwal, S. Mandalia and J. Salvado, Sterile neutrinos in astrophysical neutrino flavor, JCAP 02, 015 (2020) doi:10.1088/14757516/2020/02/015 [arXiv:1909.05341 [hep-ph]].

[44] M. Ahlers, M. Bustamante and N. G. N. Willesen, Flavors of astrophysical neutrinos with active-sterile mixing, |arXiv:2009.01253 [hep-ph]].

[45] J. G. Learned and S. Pakvasa, Detecting tau-neutrino oscillations at PeV energies, Astropart. Phys. 3, 267274 (1995) doi:10.1016/0927-6505(94)00043-3 |arXiv:hepph/9405296 [hep-ph]].

[46] M. Ahlers, private communication.

[47] G. M. Webb, Relativistic transport theory for cosmic rays Astrophys. J. 269, 319 (1985) doi:10.1086/163451

[48] O. F. Piattella, Lecture notes in cosmology, doi:10.1007/978-3-319-95570-4 |arXiv:1803.00070 [astroph.CO]].

[49] L. A. Anchordoqui, Ultra-high-energy cosmic rays, Phys. Rept. 801, 1-93 (2019) doi:10.1016/j.physrep.2019.01.002 |arXiv:1807.09645 [astro-ph.HE]].

[50] V. Berezinsky and A. Z. Gazizov, Diffusion of cosmic rays in expanding universe, Astrophys. J. 643, 8-13 (2006) doi:10.1086/502626 [arXiv:astro-ph/0512090 [astro-ph]].

[51] M. Dentler, Á. Hernández-Cabezudo, J. Kopp, P. A. N. Machado, M. Maltoni, I. Martinez-Soler and T. Schwetz, Updated global analysis of neutrino oscillations in the presence of eV-scale sterile neutrinos, JHEP 08, 010 (2018) doi:10.1007/JHEP08(2018)010 [arXiv:1803.10661[hep-ph]].

[52] S. Gariazzo, P. F. de Salas and S. Pastor, Thermalisation of sterile neutrinos in the early Universe in the $3+1$ scheme with full mixing matrix, JCAP 07, 014 (2019) doi:10.1088/1475-7516/2019/07/014 [arXiv:1905.11290 [astro-ph.CO]].

[53] M. G. Aartsen et al. [IceCube Collaboration], Characteristics of the diffuse astrophysical electron and tau neutrino flux with six years of IceCube high energy cascade data, Phys. Rev. Lett. 125, no.12, 121104 (2020) doi:10.1103/PhysRevLett.125.121104 |arXiv:2001.09520 [astro-ph.HE]]. 
[54] M. G. Aartsen et al. [IceCube Collaboration], Evidence for astrophysical muon neutrinos from the Northern sky with IceCube, Phys. Rev. Lett. 115, no.8, 081102 (2015) doi:10.1103/PhysRevLett.115.081102 arXiv:1507.04005 [astro-ph.HE]].

[55] A. A. Aguilar-Arevalo et al. [MiniBooNE Collaboration], Significant excess of electronlike events in the MiniBooNE short-baseline neutrino experiment, Phys. Rev. Lett. 121, no.22, 221801 (2018) doi:10.1103/PhysRevLett.121.221801 |arXiv:1805.12028 [hep-ex]].

[56] A. Aguilar-Arevalo et al. [LSND Collaboration], Evidence for neutrino oscillations from the observation of $\bar{v}_{e}$ appearance in a $\bar{v}_{\mu}$ beam, Phys. Rev. D 64, 112007 (2001) doi:10.1103/PhysRevD.64.112007 |arXiv:hep-ex/0104049 [hep-ex]].

[57] M. G. Aartsen et al. [IceCube Collaboration], Searching for $\mathrm{eV}$-scale sterile neutrinos with eight years of atmospheric neutrinos at the IceCube Neutrino Telescope Phys. Rev. D 102, no.5, 052009 (2020) doi:10.1103/PhysRevD.102.052009 |arXiv:2005.12943 [hep-ex]].

[58] M. Blennow, E. Fernandez-Martinez, J. Gehrlein, J. Hernandez-Garcia and J. Salvado, IceCube bounds on sterile neutrinos above $10 \mathrm{eV}$, Eur. Phys. J. C 78, no.10, 807 (2018) doi:10.1140/epjc/s10052-018-6282-2 arXiv:1803.02362 [hep-ph]].

[59] J. L. Feng, I. Galon, F. Kling and S. Trojanowski, ForwArd Search ExpeRiment at the LHC, Phys. Rev. D 97, no.3, 035001 (2018) doi:10.1103/PhysRevD.97.035001 |arXiv:1708.09389 [hep-ph]].

[60] A. Ariga et al. [FASER Collaboration], Technical Proposal for FASER: ForwArd Search ExpeRiment at the LHC, |arXiv:1812.09139 [physics.ins-det]].

[61] A. Ariga et al. [FASER Collaboration], FASER: ForwArd Search ExpeRiment at the LHC, |arXiv:1901.04468 [hepex]].

[62] H. Abreu et al. [FASER Collaboration], Detecting and Studying High-Energy Collider Neutrinos with FASER at the LHC, Eur. Phys. J. C 80, no.1, 61 (2020) doi:10.1140/epjc/s10052-020-7631-5 arXiv:1908.02310 [hep-ex]].

[63] H. Abreu et al. [FASER Collaboration], Technical Proposal: FASERnu, arXiv:2001.03073 [physics.ins-det]].

[64] S. Buontempo, G. M. Dallavalle, G. De Lellis, D. Lazic and F. L. Navarria, CMS-XSEN: LHC Neutrinos at CMS. Experiment Feasibility Study, |arXiv:1804.04413 [physics.insdet]].

[65] N. Beni et al. [XSEN Collaboration], XSEN: a $v$ N Cross Section Measurement using High Energy Neutrinos from pp collisions at the LHC, |arXiv:1910.11340 [physics.ins- det]].

[66] C. Ahdida et al. [SHiP Collaboration], SND@LHC, |arXiv:2002.08722 [physics.ins-det]].

[67] N. Beni, M. Brucoli, S. Buontempo, V. Cafaro, G. M. Dallavalle, S. Danzeca, G. De Lellis, A. Di Crescenzo, V. Giordano and C. Guandalini, et al. Physics Potential of an Experiment using LHC Neutrinos, J. Phys. G 46, no.11, 115008 (2019) doi:10.1088/1361-6471/ab3f7c |arXiv:1903.06564[hep-ex]].

[68] W. Bai, M. Diwan, M. V. Garzelli, Y. S. Jeong and M. H. Reno, Far-forward neutrinos at the Large Hadron Collider, JHEP 06, 032 (2020) doi:10.1007/JHEP06(2020)032 |arXiv:2002.03012 [hepph]].

[69] X. Chu, B. Dasgupta and J. Kopp, Sterile neutrinos with secret interactions-lasting friendship with cosmology, JCAP 10, 011 (2015) doi:10.1088/1475-7516/2015/10/011 |arXiv:1505.02795 [hep-ph]].

[70] M. Archidiacono, S. Gariazzo, C. Giunti, S. Hannestad, R. Hansen, M. Laveder and T. Tram, Pseudoscalar-sterile neutrino interactions: reconciling the cosmos with neutrino oscillations, JCAP 08, 067 (2016) doi:10.1088/14757516/2016/08/067 [arXiv:1606.07673 [astro-ph.CO]].

[71] Y. S. Jeong, S. Palomares-Ruiz, M. H. Reno and I. Sarcevic, Probing secret interactions of eV-scale sterile neutrinos with the diffuse supernova neutrino background, JCAP 06, 019 (2018) doi:10.1088/1475-7516/2018/06/019 |arXiv:1803.04541[hep-ph]].

[72] M. G. Aartsen et al. [IceCube Collaboration], Search for neutrinos from decaying dark matter with IceCube, Eur. Phys. J. C 78, no.10, 831 (2018) doi:10.1140/epjc/s10052018-6273-3 [arXiv:1804.03848 [astro-ph.HE]].

[73] G. Steigman, D. N. Schramm and J. E. Gunn, Cosmological limits to the number of massive leptons, Phys. Lett. B 66, 202-204 (1977) doi:10.1016/0370-2693(77)90176-9

[74] G. Mangano, G. Miele, S. Pastor, T. Pinto, O. Pisanti and P. D. Serpico, Relic neutrino decoupling including flavor oscillations, Nucl. Phys. B 729, 221 (2005) doi:10.1016/j.nuclphysb.2005.09.041 |hep-ph/0506164|.

[75] M. G. Aartsen et al. [IceCube Gen2 Collaboration], IceCube-Gen2: The window to the extreme universe, |arXiv:2008.04323 [astro-ph.HE]].

[76] S. Aiello et al. [KM3NeT Collaboration], Deepsea deployment of the KM3NeT neutrino telescope detection units by self-unrolling, JINST 15, no.11, P11027 (2020) doi:10.1088/1748-0221/15/11/P11027 |arXiv:2007.16090 [astro-ph.IM]]. 